\title{
Non monotonic reasoning rules for energy efficiency
}

\author{
Claudio Tomazzoli $₫$, Matteo Cristani ${ }^{\natural}$, Erisa Karafili ${ }^{\llbracket}$, and Francesco Olivieri ${ }^{\llbracket}$ \\ ${ }^{a}$ Department of Computer Science, University of Verona, Strada Le Grazie, 37100 Verona, Italy, \\ E-mail: claudio.tomazzoli@univr.it \\ ${ }^{\mathrm{b}}$ Department of Computer Science, University of Verona, Strada Le Grazie, 37100 Verona, Italy, \\ E-mail: matteo.cristani@univr.it \\ ${ }^{\mathrm{c}}$ Department of Computing, Imperial College London, 180 Queen's Gate, SW7 2AZ, London, UK \\ E-mail: e.karafili@imperial.ac.uk \\ ${ }^{\mathrm{d}}$ Data61, 70-72 Bowen St, Spring Hill, Brisbane Qld 4000, Australia \\ E-mail:francesco.olivieri@data61.csiro.com.au
}

\begin{abstract}
Conflicting rules and rules with exceptions are very common in natural language specification employed to describe the behaviour of devices operating in a real-world context. This is common exactly because those specifications are processed by humans, and humans apply common sense and strategic reasoning about those rules to resolve the conflicts. In this paper, we deal with the challenge of providing, step by step, a model of energy saving rule specification and processing methods that are used to reduce the consumptions of a system of devices, by preventing energy waste. We argue that a very promising non-monotonic approach to such a problem can lie upon Defeasible Logic, following therefore an approach that has shown success in the current literature about usage of this logic for conflict rule resolution and for human-computer interaction in complex systems. Starting with rules specified at an abstract level, but compatibly with the natural aspects of such a specification (including temporal and power absorption constraints), we provide a formalism that generates the extension of a basic Defeasible Logic, which corresponds to turned on or off devices.
\end{abstract}

Keywords: Ambient intelligence techniques, intelligent systems, knowledge representation, automatic reasoning, defeasible logic

\section{Introduction}

Energy consumption refers to the amount of energy used by an individual or organisation. Energy consumed in buildings, both for residential and commercial end users, accounts for one-fifth of the total delivered energy consumed worldwide and is expected to increase at a rate of $1.5 \%$ per year until 2040 [38]. This energy request is increasing also in those areas of the world where other energy requests have been controlled more wisely in the recent past, and it is therefore a demanding problem.

Energy consumption in buildings is a completely different scenario when compared to an industrial environment. While the latter has generally few big power draining devices that can be controlled individ- ually, in buildings we have a huge amount and variety of devices each of which bears a small contribution to the overall consumption but whose control is almost always neglected.

This lack of control leads to a huge energy waste, as can be better understood using this simple rule of thumb, as shown in Fig. 11 if we roughly divide the week in half days, we have five working active half days, or time intervals of twelve hours each, while we have nine of these intervals which can be defined "inactive" such as first mornings, nights and weekends. During these time intervals a good number of devices such as printers, vending machines, lights shall be turned off nevertheless it is common experience that they are left on. This is often neglected despite the fact that these time intervals sum up to almost two thirds of 
the overall time $\left(\frac{9}{14} \sim \frac{2}{3}\right)$, while the time at which they are probably correctly in operation is only one third $\left(\frac{5}{14} \sim \frac{1}{3}\right)$ of the week.

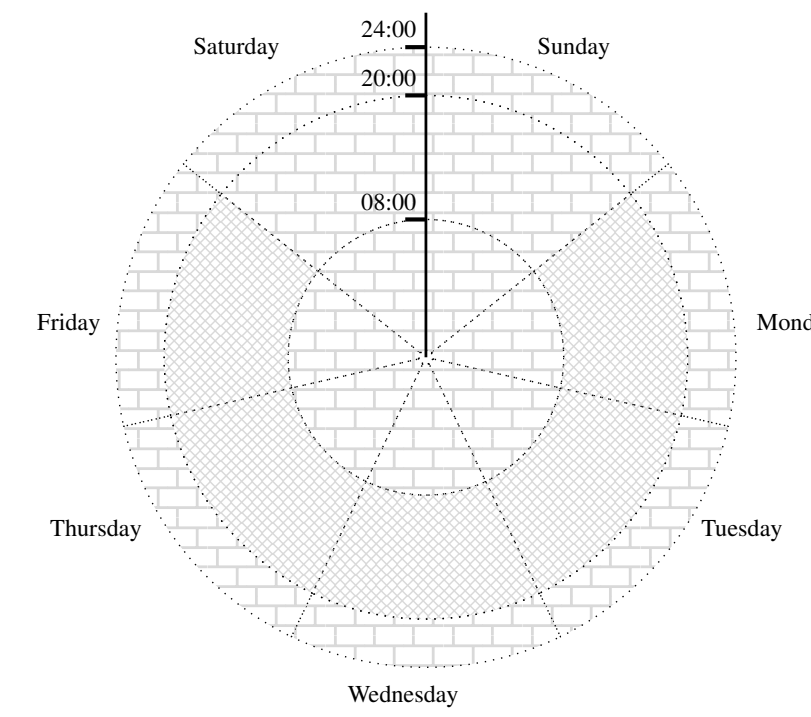

business active hours

business inactive hours

Fig. 1. Weekly energy time intervals

The problem of saving energy in buildings is strictly connected to the problem of measuring and controlling all these devices in an efficient way. The measurement and control should take into account every single state and power level, as well as the interconnection between several devices. For instance, if nobody is present in the office and it is already late evening, the vending machine in the cafeteria is wasting energy if it is still switched on, likely as much as the air conditioning in the office which should definitely be in the off state.

The above example shows how to achieve a correct energy saving. For doing that we need the help of several sensors and actuators and their interconnected data. The latter are the input for an algorithm that solves the decision problem that has as goal the energy saving as well as the various user preferences. The algorithm used during the decision process, in our opinion, needs to be fed by concepts given from a user interface. An interesting application aspect of energy saving technologies is that users often desire to regulate the behaviour of devices by means of usage rules. A usage rule is essentially a rule of the form:

\section{IF condition THEN action}

where a condition can be an instant of time, an interval of time, an absorption level, one or more devices in given specific states. On the other hand, an action corresponds to turning a device on or off, or, more generally, to execute a command of a command-based system. In this work, we do not consider devices that may have states different from on and off, as happens in real scenarios, with states like ready or stand-by. For the purpose of this paper those situations are not considered, and left to further work. In other terms, we analyse only two-phase devices, and do not consider multiphase ones.

Let us recall that the purpose of having any automation support to energy saving, in a complex device network, is to reduce the power absorbed by the system itself in any temporal instant, in order to reduce the total amount of energy consumed in any given interval. Typically, we imagine to have a general interval (that is considered uniquely), like a single year, possibly divided into sub-periods (months, weeks, days, hours and minutes), where conditions and actions are repeated.

The difficulties that emerge when dealing with problems of energy saving, with respect to the specification of usage rules for energy absorbing devices are not trivial. The usage rules can have contradictory actions for similar conditions, that consist in one of their main difficulties. These rules can naturally be represented as rules with exceptions.

For instance, consider an office where an user specifies RULE 1: Turn the lights off at 8pm. This rule should hold in general, but it is very natural to imagine an exception, like RULE 2: Do not execute RULE 1, if someone is in the office.

Another possible situation is when two rules are in conflict, possibly with an expressed priority. For instance, consider the configuration mentioned above. A user specifies RULE 3: Turn the coffee machine off, when it is 6pm, and RULE 4: Turn the coffee machine on, when someone is in the office. In this case, in particular, we can have the functionality preserving principle that brings up the priority of RULE 4 with respect to RULE 3; on the contrary, we can have the saving principle, that brings up the priority of RULE 3 with respect to RULE 4.

Rules as specified above are triggered by temporal instants (on a repeated basis) and temporal intervals e.g., in rules that are to be used only in a specific interval, especially those regarding heating and air con- 
ditioning. Moreover, we are interested in two kinds of power constraints: power limits, and power minimum constraints. The former are those constraints that govern the execution of rules within a given limit (RULE 5: Turn the lights on only if the power absorption is under $2.7 \mathrm{~kW}$ ). The latter instead correspond to rules that prevent undesired turnoffs, as in RULE 6: Turn the printer on, if the absorbed power of the system is less than $100 \mathrm{~W}$.

The typical rules specified by users for energy saving are non-monotonic. Natural ways of expressing these types of rules can be Defeasible Logic. In particular, the types of rules defined above correspond to defeasible rules (the actual usage rules), defeaters (the exceptions) and a superiority relation (priority relations between conflicting rules). There are two evident differences in terms of representation needs between basic defeasible logic and the rules we specified above: temporal constraints and power constraints. In order to manage these differences, we have two possible approaches. The first consists in enriching the defeasible logic to an extent that allows the above mentioned rule forms; the second introduces a translation method that starts from rules of a new formalism into basic defeasible logic.

To better understand the differences, we need, at first, to consider the extension of a defeasible theory, and evaluate it as a means for obtaining some sort of energy saving. In fact, an energy saving solution should answer to the following need: turn off the maximum possible number of devices (or reduce their absorption) at a single time, while preserving in a complete way the functionality of the system. Therefore, for our purpose, a solution can be a list of devices that have to be turned off, and another set of devices that have to be turned on.

Basically, a possible approach would consist in specifying a temporal Defeasible Logic, where rules are associated to constraints about energy consumptions, and modifying the deduction system to take into account the above mentioned aspects. This approach seems complete and promising, but it contains one very important drawback. A system with these modifications requires a completely different inferential apparatus, and this leads to an autonomous theoretical definition of the notions of derivation, conclusion and extension. Although this method might work appropriately for the case we are studying, it is rather cumbersome and uselessly complicated to provide such a method if the intended meaning of an extension is the above mentioned one, namely the lists of devices that should be turned on or off.

The other approach makes use of the well-known inferential apparatus of standard Defeasible Logic. This approach allows the user to specify the rules, with time and power constraints in an abstract way (we will call them"metarules"), which are translated into basic defeasible theories at given instants. It uses the concept of synchronisation, that is in fact a label elimination algorithm, able to discover which basic defeasible rules can be applied at that instant.

It is important to emphasise that the basic inferential apparatus of Defeasible Logic acts in linear time. Assume that we are able to translate the metarules into a set of defeasible rules that is limited in size with respect to the metarules number. This brings a positive impact to the system synchronization that can now be performed frequently, without serious risks for the functionality and performance of the system of devices connected to the application.

The rest of the paper is organised as follows. In Section 2. we introduce related work with respect to the energy saving problem in an ambient intelligent context. We propose in Section 3 , an innovative real system where our approach can be applied. In Section 4 we present the general picture of the system and provide a visual language: after introducing the defeasible logic formalism. We also define the visual language corresponding to the metarules and the method for the translation process. In Section 5, we provide a reference implementation that concretely makes use of the approach. Finally, Section 6 takes conclusions and explores future investigations.

\section{Related Work}

In this paper we propose an architecture that deals with conflictual rules, used for improving the electric consumptions of a generic system composed by different devices.

The problem of energy saving is a widely investigated topic [9|24 33|31]. The communication technology industry has made relevant effort on this problem [7|36], by proposing new energy saving technologies, e.g., optical IP networks [637], efficient digital signature schemes in cryptography [34], and data centre architecture and design [20].

Ambient Intelligence (AmI) technologies put a special attention on increasing the efficiency during the execution of the tasks, which can also be used for 
energy saving. One important way of executing the task with these technologies is by using planning techniques, e.g., the authors in [1] use the planning for the execution of tasks based on the hierarchical task network. The authors of [14] use planning with also the improving and learning phases, for increasing the energy efficiency in an existing technology.

Another important technique used by AmI is scheduling. Scheduling can be of different types, like static and dynamic scheduling used in [21], where the authors represent the case of a student dormitory that uses embedded agents to create an AmI environment, and that is controlled by these embedded agents according to user preferences. The static/dynamic scheduling is an optimization technique, as well as the one introduced in [12] where the authors introduced an optimized schedule mechanism that makes use of machine learning techniques.

In [15], the authors propose an improved scheduling algorithm that deals with diverse constraints for energy consumption. In this case, there is no analysis of conflictual constraints, as priority values are assigned by the user to the different constraints. In [54] the authors use the ISES/ESA algorithm [11] for the scheduling and planning in the architecture of RoboCare. This scheduling technique is also optimized by the use of monitoring of the user's behaviour.

Another approach used for improving the energy consumption is based on sociological studies. In [39], the authors describe an investigation where for decreasing energy consumption, users are involved in a learning process about energy wastes and damages to the environment are pointe out.

The analyses of energy consumption information can lead to the gathering of business-relevant data, as shown for instance in [19] through a statistical approach. The authors show the existence of a linear correlation between the income of a person/family with the consumption of electricity, and prove that a systematic governance of the wastes can effectively provide higher income availability.

The different scheduling and planning solutions for energy efficiency, encounter the problem of conflictual rules. The idea of using the Defeasible Logic mechanism to handle exceptions and conflictual rules in real life scenarios is not new. Defeasible Logic has been used during the simulation of an UAVs navigation system in urban environment [26], for solving conflictual predicates. Dealing with conflicts and exceptions is an important issue also in business processes, where modal variants of defeasible Logic [30] propose regu- lation systems. On the same topic there are many further investigations that have been carried out, that have been considered for the definition of the formalism here, incuding [22[23].

Rule-based approaches have already been employed in different areas of research where they have shown to be useful. In particular, Daniele et al. have investigated the applicability of rule-based systems for context-aware applications, an area that also comprises many real-life applications, including smart environments and Internet of Things.

A very general investigation on Rule-Based systems, and in particular on those based on Jess or Drools engines can be found in [18].

Another interesting approach has been developed in [35] by Bikakis et al., for energy saving in an ambient intelligent environment is the usage of rulebased techniques. In this work it is suggested to use an agent, either reactive or deliberative, which enforces several energy-saving policies. With the use of Defeasible Logic the authors are able to incorporate also an effective and intuitive policy management.

In [8] the authors propose four strategies for solving conflicts between rules that are context dependent, in different ambient intelligence scenarios. Their solution is based on the Multi-Context Systems paradigm, where the local knowledge of the agents is represented through context. A preference ordering is used for expressing the confidence that an agent has on knowledge imported from another agent through the information flow between agents.

In this paper we go much further than has been done in [35 8] as we provide a general method for rule processing as applied to human interfaces to smart environments, in the specific case of energy saving.

Another completely different approach, based on conflict adaptation, namely on the attempt of providing some space for context-based reasoning, has been adopted by Preuveneers in [32].

\section{A real system our approach can be applied}

In this section we introduce an existing technology, Elettra, a real life system where our approach can be applied to achieve the benefits we believe can be achieved using a non classical reasoning technique. 


\subsection{The basic system: Elettra}

Elettrd $^{1}$ is an intelligent system designed to obtain efficient power consumption and to have the instant confirmation of the results, managing "de facto" how the electricity is used in a company.

Elettra is an evocative name chosen to identify a smart system where arbitrary rules can be set to automatically reduce the waste and control the usage of electricity in one or more buildings, allowing the adoption of efficiency policies and action plans objectively measurable thanks to the presence of a monitoring system that highlights the situation before and after.

These rules do not only manage the simple on/off status of the equipments, but they can also trigger alarms and send related messages via electronic mail or text messages. These rules, set by the user, adopt criteria based on time and specific conditions based on data coming from measures of physical quantities (e.g., a peak in energy consumption or other events) ${ }^{2}$,

Elettra is able to control all related equipments, provide tools for analyzing energy consumption in detail, provide forecasts and it is easily programmable by any user. Moreover, it provides the knowledge and the certainty of electricity use in order to identify trends and their consumption profiles.

Thanks to wireless technology, no modifications on existing structure is required: an installation can be made without touching the walls of the buildings where the system is to be installed.

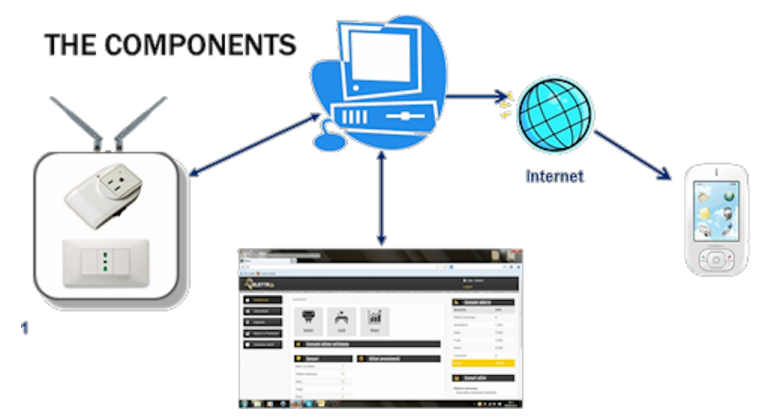

Fig. 2. Operative Concept of Elettra

Fig. 2 describes the components of the system:

\footnotetext{
${ }^{1}$ Product description can be found at http://www. risparmiarecorrente.it/?lang=en

${ }^{2}$ Reader can find an online demo at http://demoelettra. realt.it
}

- The miniserver Elettra is small and dedicated to Elettra, is pre-configured, does not occupy space and requires only a standard Internet connection;

- A radio signal collector that should be positioned next to the miniserver, also this equipment is preconfigured;

- various radioplugs that can be connected by any user, in fact just put it between the wall plug and the plug equipment you want to control (printer, monitor, lamp, refrigerator, etc..) and the same will provide to communicate each other wirelessly.

For its versatility and simplicity it is used by a wide range of people: energy managers, purchasing manager, chief executive officers of small-medium entities, and in general people in companies interested in energy saving policies.

After authentication, the user faces a dashboard (see top side of Fig. 3) in which the system summarizes daily consumptions, which sensors are active and last actions performed by the system.

There are three main menu choices: Automation, Structure and Measures. Through Automation the user can set up rules of switching on/off of equipment based not only on the operations chronology (like: night day) but also on consumption, Actions (switch on, switch off, notice) and Scenarios which are set of actions to be performed when a certain rule is fired. A rule is "fired" when certain conditions, specified in Rules, are matched.

Through Structure, the user can tell the system how the plant is made, so Buildings and Rooms can be defined as well as Sensors and Devices.

From Sensors and Measures, a list is displayed from which physical devices can be manually switched on and off remotely (see bottom side of Fig. 3). Through Measures the user can obtain reports on the actual energy consumption which allows quick and correct decisions.

Real time data are reproduced as well as historical and forecasting data of every single device of the structure and of the various elements, such as the overall consumption of all equipments in a room or even of an entire building.

The Elettra system is made of several components, both hardware and software, among these there should be mentioned:

ELETTRA_UI provides the basic web structure of the system; 
ELETTRA_CORE is an enterprise level system for measurement and control of electric consumption, from multiple building to single plug level;

ELETTRA_APP is a multi-platform "app" for mobile management of some of the features.

The overall logical architecture, as shown in Fig. 4 realises the integration between these systems and allows future evolution of single component without affecting the global system.

There is also a sub-system responsible for the communication with all devices which is modular, meaning that has a common framework and several specialised components able to deal with manufacturer specific

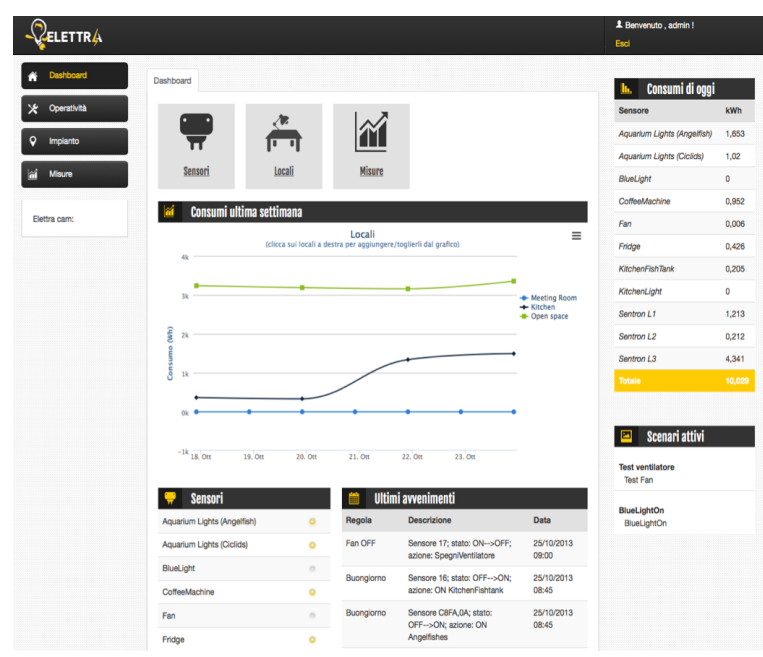

(a) Dashboard

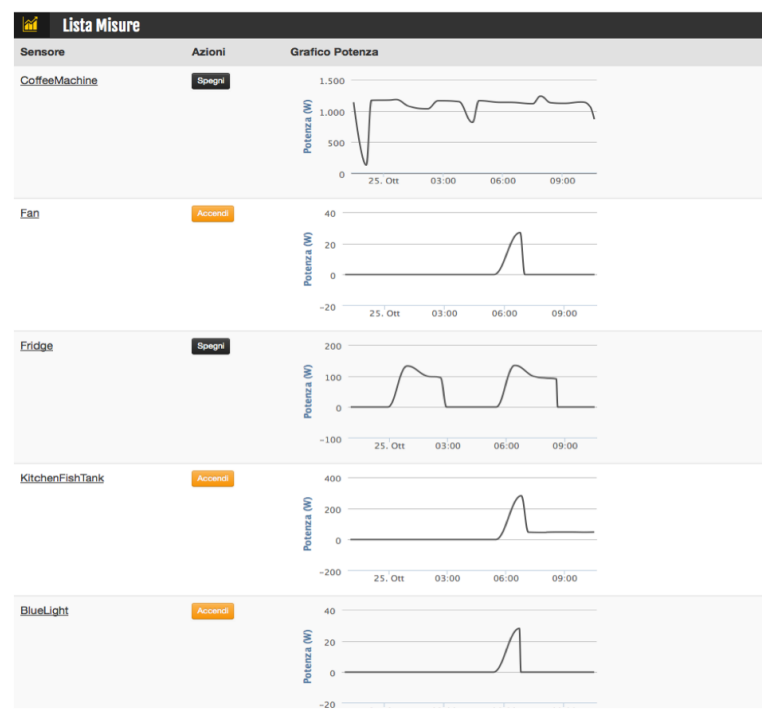

(b) Measures

Fig. 3. Screenshots of Elettra protocols. With this sub-system, new hardware such as smart plugs or industrial measurers can be integrated to the global system effortlessly. Elettra relies on the

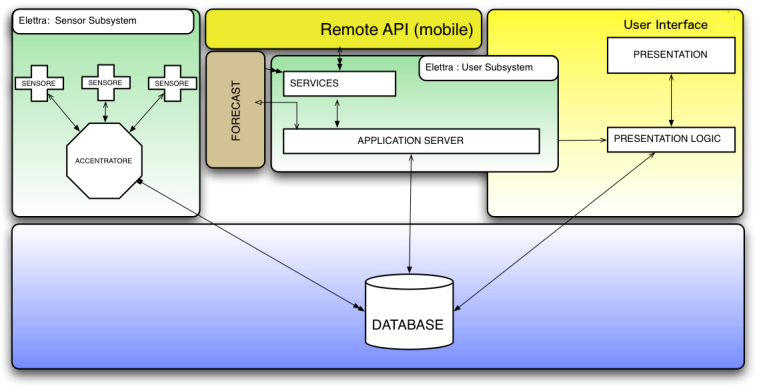

Fig. 4. Logical Model

user and his experience to achieve efficiency in energy consumption. The user is asked to define which device is attached to each sensor and is also asked to set up rules that are effective for energy saving.

This is sometimes a task for very skilled technical people and is always time consuming, especially in complex environments. Nevertheless, overall system performances rely mainly on the correct formulation and application of efficient rules, which may differ from place to place. Therefore, a way to improve the whole system is to find a possible automation where the system recognizes different devices, their consumption and how rules can be derived and applied.

\section{An architecture for energy saving based upon non-monotonic logic}

In this section, we introduce an approach to implement a method for energy saving. Such an approach uses non-monotonic reasoning about rules that specify to turn on or off a particular device. The basic idea is to represent these rules on a twofold basis: as rules specified by the user (called metarules), and as concrete defeasible rules for an actuator. The idea behind this model is that the user specifies a behavioural rule, namely she describes a condition which triggers an action, to be considered in order to prevent excessive energy consumption. We then show that the metarules can be synchronized in linear time: we translate the metarules into defeasible rules, and then pass them to an actuator.

The entire architecture is specified below. First of all, we introduce the intended meaning of the elements 
of the language. This architecture is specified in details in Section 4.6

The logic we specify in this section is two-layered. The first layer is provided in forms of metarules. Consider a usage rule specified as If a light in the corridor turns on, then turn on the lights in the whole corridor, only between 9 am and 8pm, that aims at reducing the need for lights during daylight time period. Clearly, the rule is applied (is valid) during the interval between $9 \mathrm{am}$ and $8 \mathrm{pm}$, and it is inactive during the rest of the day. If we synchronise the rule with a clock, we have that the rule is transformed into a basic defeasible rule, without time and energy constraints, only when the instant of synchronisation occurs within the interval $9 \mathrm{pm}$ to 6 am.

We now introduce the formalism. Letters represent the concept of a certain value of physical dimension such as power level being absorbed in an electric load or a light sensor reaching the level of normal lightning or again the power consumption of a fridge going above the zero threshold meaning that it is $O N$. Literals represent the concept that this certain value of physical dimension has taken place, meaning, for instance, with power consumption that the power level indicated has been absorbed, or the light level has been measured. Then, we have a set of "metarules" that will be rewritten as rules adhering to a defeasible theory thanks to a synchronisation and decision algorithm.

\subsection{Metarules}

Metarules will be written with two constraints: time and measure. In this context, measures are going to be power absorption. We have rules which are based only on time, and rules which depend on a certain measure to cross a threshold upward or downward. These rules are written in form of defeasible rules, extended with a label $\left[t_{1}, t_{2}\right]$ to be put before the letters and criteria based on measures are labels in the form $p+$ or $p$ above the derivation operation sign.

The syntax is defined as follows. Well-formed formulae are

$$
\begin{aligned}
& \text { (1) } r,\left[t_{1}, t_{2}\right]: a_{1}, \ldots, a_{n} \Rightarrow b, n \geq 0 \\
& \text { (2) } r,\left[t_{1}, t_{2}\right]: a_{1}, \ldots, a_{n} \stackrel{p+}{\Longrightarrow} b, n>0 \\
& \text { (3) } r,\left[t_{1}, t_{2}\right]: a_{1}, \ldots, a_{n} \stackrel{p-}{\Longrightarrow} b, n>0 \\
& \text { (4) } r_{1} \succ r_{2}
\end{aligned}
$$

with the following meanings, given that a positive literal $a$ will always mean that the corresponding device is $O N$ while $\neg a$ would mean that the device is $O F F$
(1) $r,\left[t_{1}, t_{2}\right]: a_{1}, \ldots, a_{n} \Rightarrow b$ is a rule and means that at time between $t_{1}$ and $t_{2}$ if $a_{1}, \ldots, a_{n}$ are $O N$ then $b$ is $O N$.

(2) $r,\left[t_{1}, t_{2}\right]: a_{1}, \ldots, a_{n} \stackrel{p+}{\Longrightarrow} b$ is a rule and means that at time between $t_{1}$ and $t_{2}$ if $a_{1}, \ldots, a_{n}$ are over $p$ then $b$ is $O N$.

(3) $r,\left[t_{1}, t_{2}\right]: a_{1}, \ldots, a_{n} \stackrel{p-}{\Longrightarrow} b$ is a rule and means that at time between $t_{1}$ and $t_{2}$ if $a_{1}, \ldots, a_{n}$ are $u n$ $\operatorname{der} p$ then $b$ is $O N$

(4) $r_{1} \succ r_{2}$ mean that rule $r_{1}$ prevails over rule $r_{2}$.

Note that when $n=0$ we have a rule with no antecedents, that forces a device to be turned on or off.

For instance, we can write:

I $[08: 00,18: 00]$ : fridge meaning that at time between 08 and 18 the fridge is $O N$, while

I $[08: 00,18: 00]: \neg$ fridge means that at time between 08 and 18 the fridge is $O F F$.

【 $[08: 00,18: 00]:$ fridge $\stackrel{700+}{\longrightarrow} \neg$ oven means that at time between 08 and 18 if the fridge is over 700 Watt than the oven should be OFF.

We can then express simple such statements such as "after 8pm switch off the coffee machine"

】 [20:00, 24:00] : $\Rightarrow \neg$ coffemachine

and also powerful rules such "after $8 \mathrm{pm}$ switch off the coffee machine unless the computer is working" which we rewrite as "after $8 \mathrm{pm}$ if the computer is using less than 10 (Watt) then switch off the coffee machine":

\section{】 $[20: 00,24: 00]:$ computer $\stackrel{10-}{\Longrightarrow} \neg$ coffemachine.}

More precisely, we have two types of rules. Those expressing states of devices (e.g., at a given time, or in a given interval, the fridge should be turned on), and those that provide conditions (on or off devices), validity intervals (not mandatorily both, with intervals that can be substituted by single instants) and power constraints (minimum and maximum), deriving desirable states of one device.

Generally speaking, the system we are devising here, is a clausal machinery. In clausal machineries, no logical disjunctions are provided, as the conjunction of clauses with the same consequence are logically equivalent to clauses with disjunction of the antecedents and consequence the common one. In the view of improving the user experience, it would, however be beneficial to provide such a mechanism. We then define a way of expressing a group of metarules with the meaning of disjunction among the antecedents, and we use the + sign to separate elements in the antecedent: 


$$
\text { 【 } r,\left[t_{1}, t_{2}\right]: a_{1}+\cdots+a_{n} \stackrel{p-}{\Longrightarrow} b, n>0
$$

which is only a compact way of writing a set of rules and therefore has the meaning of the below:

$$
\begin{aligned}
& \text { 】 } r_{1},\left[t_{1}, t_{2}\right]: a_{1} \stackrel{p-}{\Longrightarrow} b \\
& \text { ป } \ldots \\
& \text { 】 } \ldots \\
& \text { 】 } r_{n},\left[t_{1}, t_{2}\right]: a_{n} \stackrel{p-}{\Longrightarrow} b .
\end{aligned}
$$

This method is a convenient one for expressing rules in a compact way. Before describing the rewriting rules, we make the reader familiar with few standard concept of Defeasible Logic.

\subsection{Defeasible Logic}

The aim of this section is to give some basic notions of the Defeasible Logic apparatus (DL) [28]3]. DL is typically sceptical, meaning that it allows rules for opposite conclusions. In the situation where rules for opposite literals are activated (may fire), the logic does not produce any inconsistency but it simply does not draw any conclusion unless a preference (or superiority) relation states that one rule prevails over the other. A defeasible theory $D$ is defined as a structure $(F, R, \succ$ ), where (i) $F$ is a set denoting simple pieces of information that are considered to be always true (e.g., a fact is that "the stove is ON", formally stove_ON), (ii) $R$ contains two finite sets of rules: defeasible rules and defeaters, (iii) $>\subseteq R \times R$ is a binary relation. A rule is an expression $r: A(r) \hookrightarrow C(r)$, and consists of: (i) a unique name $r$, (ii) the antecedent $A(r)$ which is a finite set of (modal) literals, (iii) an arrow $\hookrightarrow \in\{\Rightarrow, \sim\}$ denoting, respectively, defeasible rules and defeaters, and (iv) its consequent $C(r)$ which is a single literal (or a chain of modal literals, see below). A defeasible rule can be defeated by contrary evidence; for example the rule "working_hours $\Rightarrow$ coffeeMachine_ON", means that during the working hours, the coffee machine is typically on, unless there is evidence proving otherwise. Defeaters are special rules whose only purpose is to defeat defeasible rules by producing contrary evidence. Our framework does not use strict rules or defeaters, but only defeasible rules.

The superiority relation $\succ$ is used to define when one rule may override the conclusion of another one, e.g., given the rules

" $r:$ John_working $\Rightarrow$ coffeeMachine_ON" and

" $s$ : S unday $\Rightarrow \neg$ coffeeMachine_ON", if we state that " $s \succ r$ ", then, unfairly, even if John is working on Sunday, he cannot use the coffee machine since it is turned off. (The infix notation $s \succ r$ means that $(s, r) \in \succ$.)

Since the authors use superiority relation there is no need for defeaters because it has been shown that the latter can be effectively substituted with the use of superiority relation.

Like in [3], the authors consider only a version of this logic that can be reduced to a propositional theory. In particular they do not take into account function symbols and quantifiers. Expressions with variables represent the finite set of variable-free instances. Moreover, in the same work, authors proved that it is always possible to remove defeaters from the theory.

A defeasible conclusion is a tagged literal and can have one of the following form:

- $+\Delta q$, which means that there is a definite proof for $q$ in $D$; such a proof uses strict rules and facts only.

- $-\Delta q$ which means that $q$ is not definitely provable in $D$.

- $+\partial q$ which means that $q$ is defeasibly provable in D.

- $-\partial q$ which means that $q$ is refuted, or not defeasibly provable in $D$.

Literal $q$ is definitely provable if either (1) is a fact, or (2) there is a strict rule for $q$, whose antecedents have all been definitely proved.

Positive and negative proof tags are related by the principle of strong negation. This is closely related to the function that simplifies a formula by moving all negations to an inner most position in the resulting formula, and replaces the positive tags with the respective negative tags, and the other way around [2]. Hence, we does not show the negative proof tags.

The following statements define notions of being applicable and discarded. In the proof condition for $\pm \partial$, a rule $r \in R_{s d}$ is (i) Applicable iff $\forall a \in A(r)$, $+\partial a \in P(1 . . n)$; (ii) Discarded iff $\exists a \in A(r)$ such that $-\partial a \in P(1 . . n)$.

We now introduce proof tags for the defeasible provability.

$+\partial$ : If $P(n+1)=+\partial q$ then

(1) $+\Delta q \in P(1 . . n)$ or

(2) $(2.1)-\Delta \sim q \in P(1 . . n)$ and

(2.2) $\exists r \in R[q]$ s.t. $r$ is applicable, and

(2.3) $\forall s \in R[\sim q]$. either $s$ is discarded, or (2.3.1) $\exists t \in R[q]$ s.t. $t$ is applicable and $t \succ s$. 
The idea of $+\partial$ derivation being that a given literal $q$ is defeasibly provable if either it is definitely provable, or we argue by using the defeasible part of the theory. In the latter case, $\sim q$ must be not definitely provable, and there must exist an applicable strict or defeasible rule for $q$. Finally, every attack on such a rule is either discarded, or defeated by a stronger rule supporting $q$.

\subsection{Rewriting the rules}

Our system works on a synchronisation basis. A clock counts discretely time ever since, and a synchroniser checks rules for eligibility of rewriting at every clock increase.

The process of rewriting is twofold. On one hand, when the defeasible rules derived from the metarules prove a given literal, which is independent of the states of the devices in the system: the intended meaning is that the device should be in the state expressed in the literal (on or off). Conversely, the rules also apply to the state of the devices, since certain rules can only be used when the devices are in a given state. Therefore, we should consider the former as a revision request, whilst the latter is a deductive closure request. We assume that the reasoner computes the above described cycle of computation (closure, revision by merge, closure).

We introduce a rewriting algorithm (Algorithm 1 . SincroCut) that takes as input the metarules and the states of the devices, and transforms them onto a defeasible theory by checking the power and temporal constraints as described below. The result of the Algorithm SincroCut is a defeasible theory. As widely analysed in the literature of defeasible logic, a defeasible theory derives four sets of literals, called, respectively positive strict extension, negative strict extension, positive defeasible extension and negative defeasible extension. Once we have provided the translation of the metarules into defeasible rules, a technique for computing extensions applies that gives out the fours sets aforementioned. As illustrated in Section 4.6 we have implemented this part with standard defeasible processing techniques.

The algorithm recurs on the function decideActiveDevice that takes as input a measure on a given device and returns a literal (positive or negative) asserting the state of the corresponding device. The first cycle of the algorithm computes the facts in the theory. The second cycle of the synchroniser, which we will also call transformer reads metarules and priorities and puts them in the sets of the theory.
Every metarule is described as $r$, the temporal labels by $r . t_{1}$ and $r . t_{2}$, the antecedents by $A(r)$ and the consequent by $C(r)$, the power constraint value is $r . p$, and the flag r.flag, true for less than or equal constraints, false otherwise. The procedure eval is used to compose the values of the antecedents in terms of current power absorption. It takes a set of literals and checks the power absorption of them in the environment at the current moment. The rules in the set of defeasible rules are simply represented by records with antecedents and consequent. The procedure createNewRule creates a new empty rule.

In the implementation of the system, we are employing a desription of rules in a very simple data structure. In other terms, when passed to the algorithm, rules and superiority relations are typed. When they are superiority relations the function type returns priority, otherwise it is a defeasible rule. Priorities are just added as they are, since they indeed coincide syntactically with the superiority relations of defeasible logic.

A certain rule is a candidate for rewriting, only if the synchroniser acknowledged that its clock time falls within the validity interval of the rule (if the rule has a validity interval) or at the exact instant of the rule if the rule is simply instantaneous.

We now show how the algorithm works with the following example.

[08: 00,18:00] : fridge $\stackrel{700+}{\Longrightarrow} \neg$ oven is a "meta rule" which will be rewritten as fridge $\Rightarrow \neg$ oven which is a common defeasible rule.

\subsection{Resulting rules}

Once the method of the algorithm provided in Subsection 4.3 is applied, the rules resulting by the rewriting are basic defeasible rules, and can be treated as shown in Section 4.2. Below, we provide an example of the rewriting process.

- + - $f$ ridge deduces that the fridge at time $t$ should be in state $O N$ hence that the controller, activated by the reasoner, will send the command to switch the fridge on.

- $+\partial \neg$ fridge deduces that the fridge at time $t$ should be in state $O F F$ thus that the controller, activated by the reasoner, will send the command to switch the fridge off.

- - fridge deduces that the fridge at time $t$ cannot be said if it shall be $O N$ or $O F F$, hence the controller will remain inactive. 


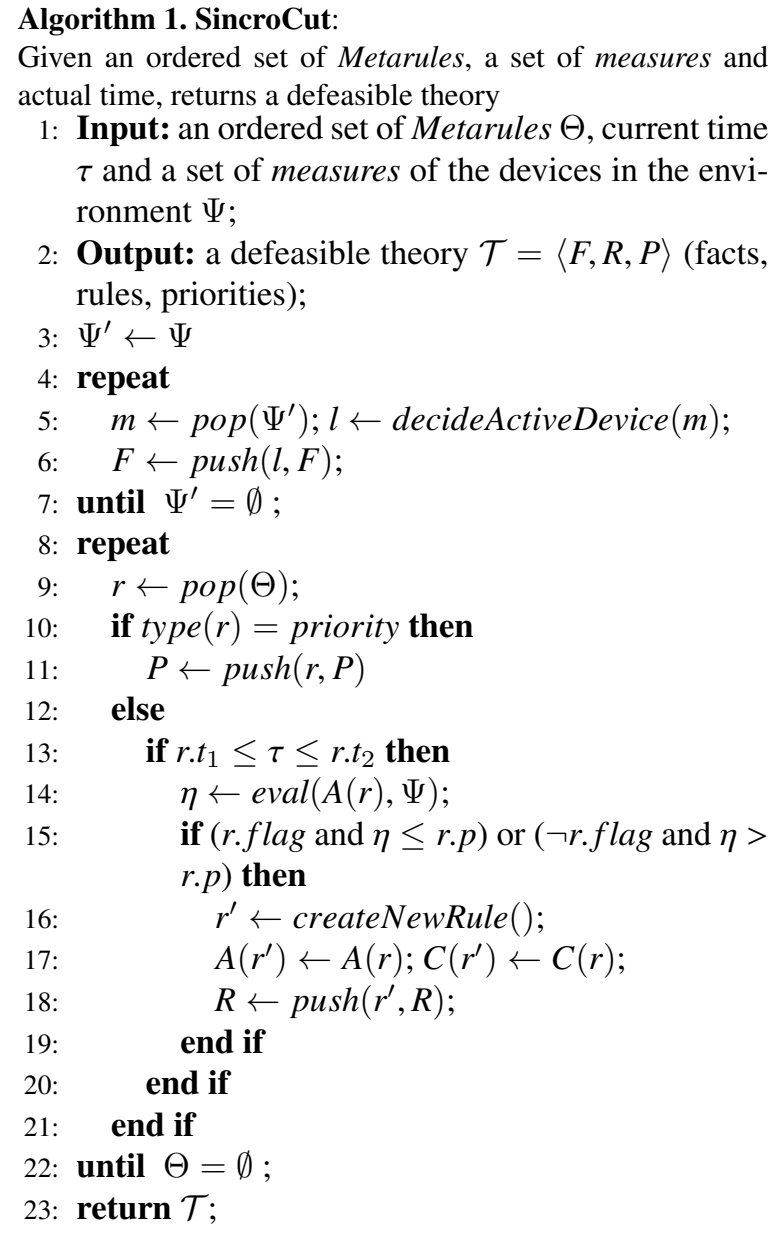

\subsection{Visual Language}

In this section we define a visual language so that the user interface can be used with little technical knowledge.

Investigations have already been carried out so far that aim at providing models for visual representation of rules. In particular Nute et al. [29] have deeply investigated the notion of defeasible logic logic graphs a visual tool to represent defeasible logic. Further on, in [25] the idea of rules as tools for managing reasoning in a visual fashion has been investigated. The FUJABA tool suite, as studied in [10] gives a general environment to deploy defaults, and potentially default reasoning and defeasible logic.

The limit of those tools that have brought us to the idea of developing a novel model lies entirely in the simplicity of the adopted strategy, that aims at providing an interface that allows to provide specifications without the limits of the previously introduced ones, that are quite oriented to the generality of the framework and also very focussed on the precision of the formalism. The idea is instead to provide a framework that, above its simplicity, also results sufficiently specific to limit itself in some elementary functions, preventing the risk of a visual language that is too complex to be used by unspecialised users.

Our language will have only a few symbols, defined in Fig. 5. We will have symbols for

Element, with attributes such as object_name, time, physical_quantity to represent a literal in our metarule.

Condition, with attributes such as value, physical_quantity, sign where a + sign represents that the condition is matched when the value of the assumptions goes "above" the value while a - sign represents that the condition is matched when the value of the assumptions goes "below" it.

along with junction lines and filled circle to represent the joining of elements.

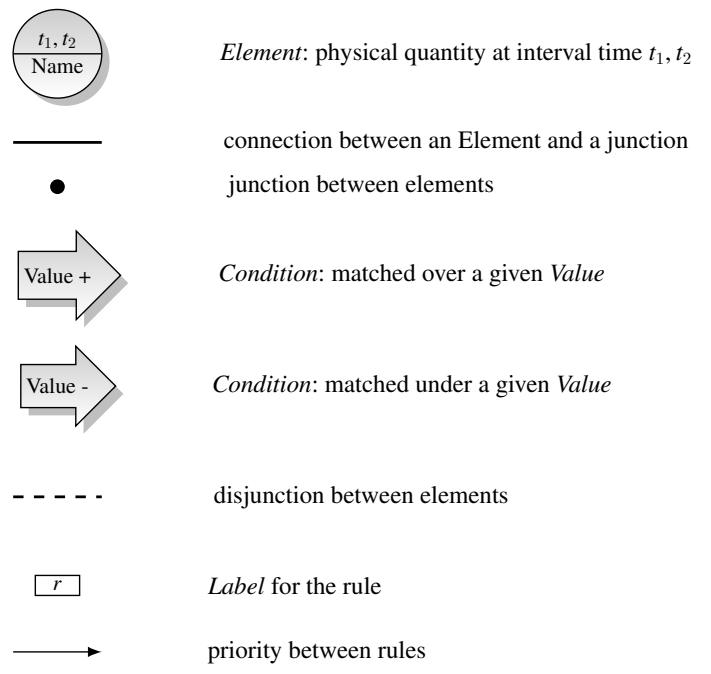

Fig. 5. Visual Language for metarules.

As junctions are used for conjunction, a disjunction sign has the meaning of $O R$ and will be translated in a metarule with the + sign between elements, whereas the junction will lead to a comma separated list of elements. The disjunction sign can be used only in the left part of the visual rule, equivalent to the antecedent of the corresponding metarule.

The time constraint of an Element can be represented with only one value $t_{1}$, assuming that this means that $t_{2}$ is midnight or $12 \mathrm{pm}$ (24:00). Thus, a visual rep- 
resentation of a rule might be simple to draw and to read, as shown in Fig. 6

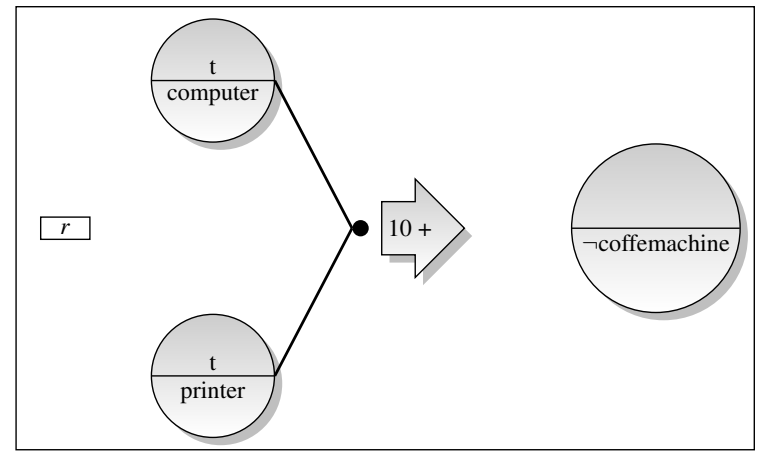

Fig. 6. Visual Example of a Metarule: at time t, if computer and printer are above 10 Watt then the coffee machine shall be off.

Rules can have also a priority, namely can be related by superiority relations, which is rendered with an arrow connecting the label of rule $r_{1}$ and rule $r_{2}$, with the direction of the arrow expressing which rule has priority over the other, so that $r_{1} \succ r_{2}$ will be rendered as
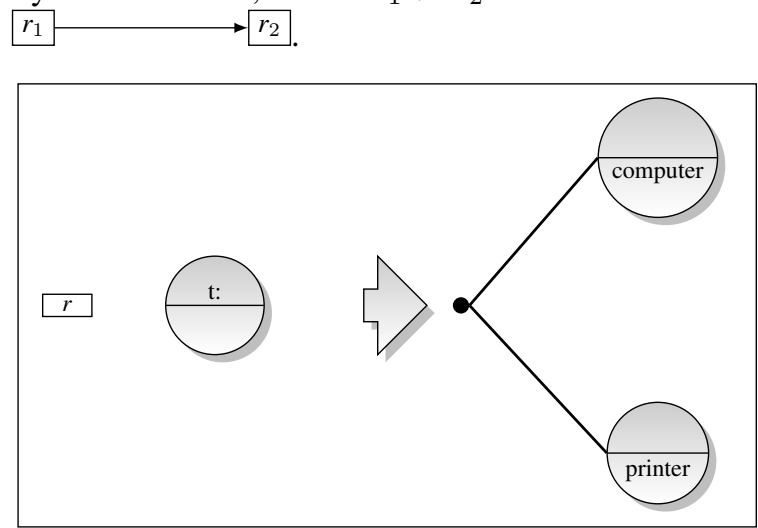

Fig. 7. Visual Example of a Metarule with common assumptions: at time $t$, computer and printer shall be on.

Rules with common assumptions can be combined in a single visual representation in which there will be a single assumption and several conclusions joined with the junction lines and circle, as shown in Fig.7.

A special meaning is associated with a "NULL" value for the name_of_the_object, meaning that the element represents a certain moment in time regardless of anything else. Analogously, a "NULL" value for the time, means that the element represents a certain value of a physical dimension, in our case, absorbed power, regardless of time. Finally, a "NULL" value is bound for a condition, meaning that the condition is always matched.

\subsection{Architecture}

Our reference architecture is made of several modules, each one with a single responsibility; the logic model of which is reported in Fig. 8

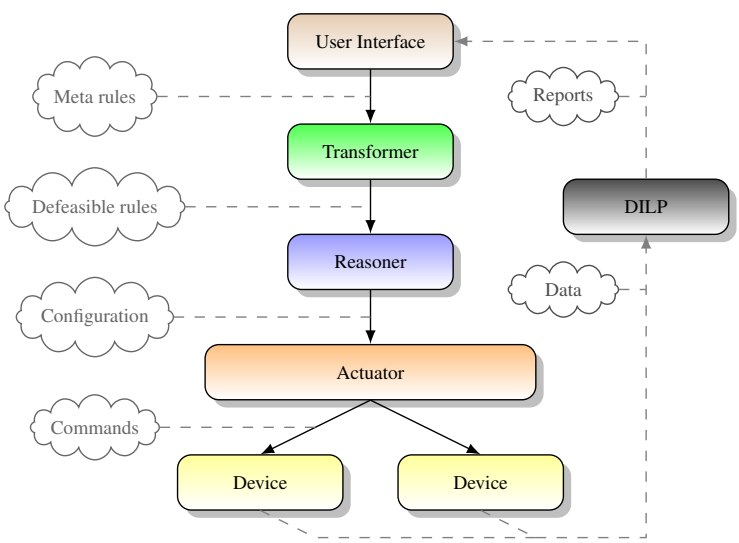

Fig. 8. Logic model of reference architecture

Every module is related to at least one other:

User Interface: allows the user to input "meta rules" and provides visualization of all data coming from the DILP module;

Transformer: takes as input a set of "meta rules" and gives as output a set of defeasible rules to be used by the Reasoner, according with time given by its internal clock mechanism and an evaluation algorithm;

Reasoner: uses the rules and determines the "should be" configuration of the system, meaning which device shall be active and at which level;

Actuator: is responsible for the delivery to the devices of all commands which are compliant with the desired configuration;

Device: deals with real devices, delivering commands and collecting data;

DILP: gathers data and makes analysis delivering summaries and possible rules to be displayed by the User Interface (future extension).

The output of the User Interface is an ordered set of "Meta rules" which are one of the input of the Transformer which runs continuously and at given times uses Algorithm 1 to produce a set of defeasible rules.

These rules are to be given to the Reasoner whose output is the using $+\partial$ conclusion given the set of rules coming from the Transformer a set of devices with their "should be" state which is called a configuration to be put in place at that time. The defeasible logic rule 
engine we used is an engine, Spindle [27], which has the benefit, among others, of effortless integration with the system Elettra being both based on a Java language program.

At different times different configurations are possible, due to the work of the Transformer.

The configuration is then the input for the Actuator whose output is a set of commands specific for each Device which in turn executes these commands delivering the physical command to the physical device. A device whose state has not been derived by the $+\partial$ conclusion of the Reasoner will be left in their previous state.

As explained in Section 6, data are gathered and interpreted to extract possible rules to be delivered as "hints" to the user. This is the responsibility of the DILP which act as feedback generator for the whole system, enabling the possibility for the system to achieve continuous enhancement of performances.

\section{Reference Implementation}

We would like to use an example from everyday experience to better show how our model can fit a real life scenario.

We consider an ambient like a small office as a stereotype of environment in which energy consumption occurs and we limit our investigation to electrical energy for the sake of simplicity, knowing that the same reasoning can be applied easily to other types of energy such as heating.

We consider a small office, as shown in Fig. 9. We decided to work with a small office because a bigger one, from the energy consumption point of view, can be modeled as the composition of a number of small offices. The representation in Fig. 9 is actually coming from the real word, being the headquarter of the company in Verona, Italy, which is the producer of the Elettra system (see Section 3).

Technological plants in industrial production need a more complex modeling, but with a higher level of abstraction can be viewed as environments in which several different devices operates together, such as in a small office. Nevertheless, a small office can represent also a good approximation of a flat in which a family lives.

A typical configuration in an office usually comprises:

- A few Computers;

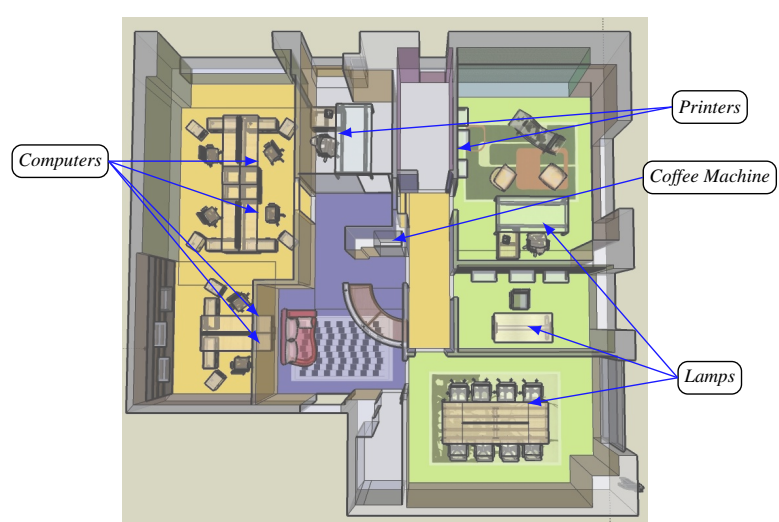

Fig. 9. A real world office layout

- One or more Printers:

- At least one Coffee Machine;

- A few Lamps;

- Ceiling lightning.

Within this office, to achieve a saving in electric energy consumption, the power consumption level of these devices shall be monitored, rules should be defined and actions based on the rules should be performed. Simple rules that can be put in action are, for instance, the ones which are in use in the office of this company:

(1) At $\mathbf{1 0}$ am switch off the lamps: there is probably no need because of ambient light is more than enough to work comfortably;

(2) At 8 pm switch off the printers: because after 8 o'clock nobody is probably working, so printers are unnecessary;

(3) At $8 \mathrm{pm}$ do not switch off the printers if at least one computer is on: because after 8 o'clock nobody is probably working, thus printers are unnecessary, unless there is a computer working in which case probably someone is there working and maybe the printer can be needed;

(4) At 10 pm switch off the coffee machine if printers are off but do not do it if at least one computer is on: because after 10 o'clock nobody is probably working and the printers are all off, hence coffee machine is not to be used, unless there is a computer working, in which case probably someone is there working and maybe the coffee machine can be used;

(5) At 7 am switch on the coffee machine and the printers: because in a while the office will be in full operational and everything shall be on and running. 
To implement these rules, only a few conceptual steps are to be done. Devices shall be named as $\left\{c_{1}, c_{2}, c_{3}, c_{4}\right\}$ for computers, $\left\{l_{1}, l_{2}, l_{3}\right\}$ for lamps, $\left\{p_{1}, p_{2}\right\}$ for printers, and $\left\{v_{1}\right\}$ for the Coffee Machine. The threshold should be defined so that we can design visual rules similar to the one show in Fig. 10 for rule number one.

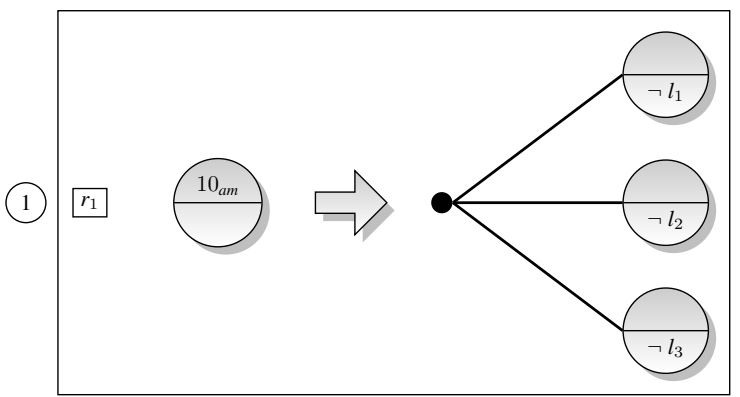

Fig. 10. Visual rule for rule one of the reference implementation.

The visual representation of rule two is similar to visual rule one as that of rule five, with the only difference of the sign meaning "ON" or "OFF", so that they will not be given here for the sake of space.

We can write also the visual representation of rule number three as show in Fig. 11, taking into account the elements of our language. "at least one computer is on" means that the consumption of at least one computer is above zero. When reading the rules as they are

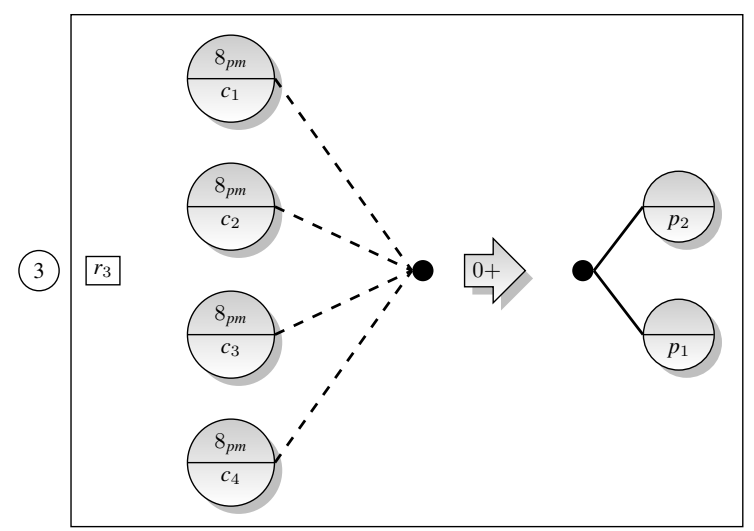

Fig. 11. Visual rule for rule three of the reference implementation.

expressed in natural language, a priority between rule number three and rule number two can be easily deduced, so that in the visual representation these rules are to be connected with an arrow, as shown in Fig.12

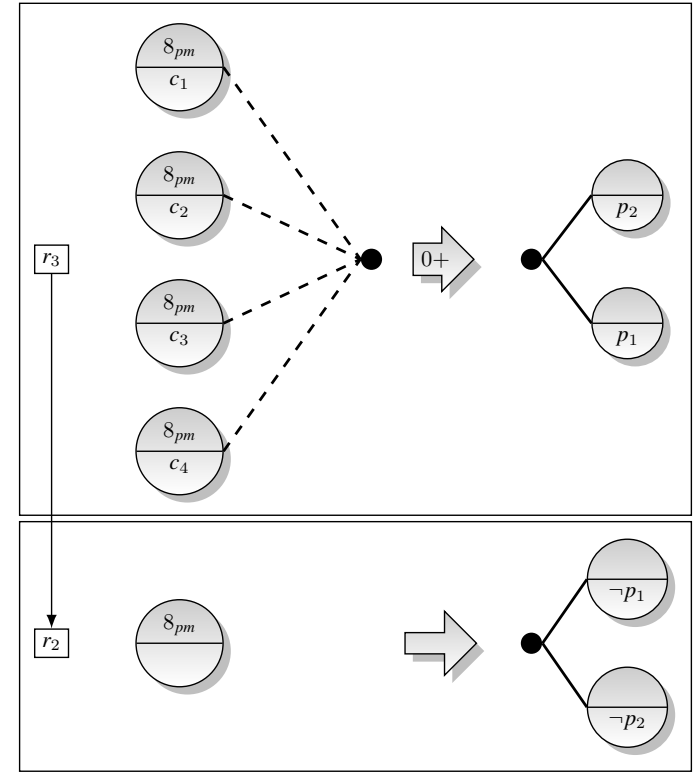

Fig. 12. Visual priority between rules

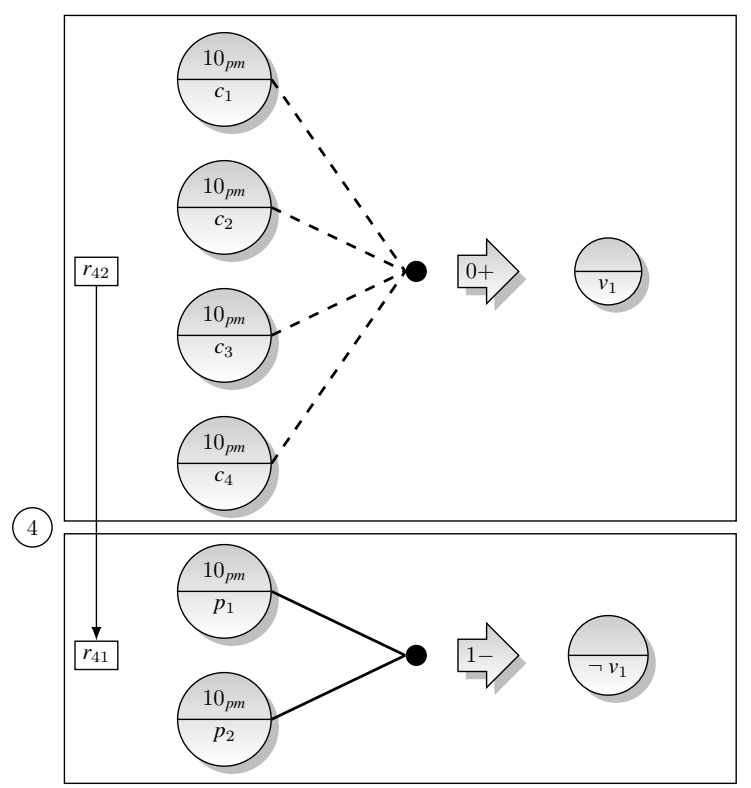

Fig. 13. Visual rule for rule four of the reference implementation.

We can write also the visual representation of rule number four, as show in Fig. 13

For the sake of readability it is strongly suggested to place the rule which prevails visually above the other, as shown in Fig. 13] where rule $r_{42}$ that has a priority is over rule $r_{41}$

Given this visual representation of aforementioned behavioural rules $\{1,2,3,4,5\}$, the corresponding 
"meta rules" to these visual rules will be expressed as follows:

$$
\text { (1) } \begin{aligned}
& r_{11}\left[10_{a m}, 12_{p m}\right]: \Rightarrow \neg l_{1} \\
& r_{12}\left[10_{a m}, 12_{p m}\right]: \Rightarrow \neg l_{2} \\
& r_{13}\left[10_{a m}, 12_{p m}\right]: \Rightarrow \neg l_{3} \\
& \text { (2) } r_{21}\left[8_{p m}, 12_{p m}\right]: \Rightarrow \neg p_{1} \\
& \\
& r_{22}\left[8_{p m}, 12_{p m}\right]: \Rightarrow \neg p_{2} \\
& \text { (3) } r_{31}\left[8_{p m}, 12_{p m}\right]: c_{1}+c_{2}+c_{3}+c_{4} \stackrel{0+}{\Longrightarrow} p_{1} \\
& r_{32}\left[8_{p m}, 12_{p m}\right]: c_{1}+c_{2}+c_{3}+c_{4} \stackrel{0+}{\Rightarrow} p_{2}
\end{aligned}
$$

Because it has been visually specified $r_{3} \succ r_{2}$ this will lead to a prioritisation when the rewriting algorithm is applied, so that it will be $r_{3} \succ r_{2}$.

(4) $r_{41}\left[10_{p m}, 12_{p m}\right]: p_{1}, p_{2} \stackrel{1-}{\Longrightarrow} \neg v_{1}$ $r_{42}\left[10_{p m}, 12_{p m}\right]: c_{1}+c_{2}+c_{3}+c_{4} \stackrel{0+}{\Longrightarrow} v_{1}$. Because it has been visually specified $r_{42} \succ r_{41}$ this will lead to a prioritisation when the rewriting algorithm is applied, so that it will be $r_{42} \succ r_{41}$.

(5) $r_{51}\left[7_{a m}, 12_{p m}\right]: \Rightarrow v_{1}$

$r_{52}\left[7_{a m}, 12_{p m}\right]: \Rightarrow p_{1}$

$r_{53}\left[7_{a m}, 12_{p m}\right]: \Rightarrow p_{2}$

We can then consider for this office a measure vector at time $\tau$ as

$p_{\tau}=\left\{m_{c_{1}}, m_{c_{2}}, m_{c_{3}}, m_{c_{4}}, m_{l_{1}}, m_{l_{2}}, m_{l_{3}}, m_{p_{1}}, m_{p_{2}}, m_{v_{1}}\right\}$ in which the elements are power consumptions of the corresponding device, expressed in Watt.

So if for instance at $11_{p m}$ the measure vector is $p_{11_{p m}}=\{100,0,0,0,0,0,0,0,0,0\}$, after the Translator has done its elaboration we can have:

【 $c_{1} t_{0}, \sim c_{2} t_{0}, \sim c_{3} t_{0}, \sim c_{4} t_{0}, \sim l_{1} t_{0}, \sim l_{2} t_{0}, \sim l_{3} t_{0}$,

【 $\sim p_{1} t_{0}, \sim p_{2} t_{0}, \sim v_{1} t_{0}$

$\begin{array}{llll}r_{11} & : \Rightarrow \neg l_{1} & r_{12} & : \Rightarrow \neg l_{2} \\ & & r_{13} & : \Rightarrow \neg l_{3} \\ r_{21} & : \Rightarrow \neg p_{1} & r_{22} & : \Rightarrow \neg p_{2} \\ r_{31_{1}} & : c_{1} t_{0} \Rightarrow p_{1} & r_{32_{1}} & : c_{1} t_{0} \Rightarrow p_{2} \\ r_{31_{2}} & : c_{2} t_{0} \Rightarrow p_{1} & r_{32_{2}} & : c_{2} t_{0} \Rightarrow p_{2} \\ r_{31_{3}} & : c_{3} t_{0} \Rightarrow p_{1} & r_{32_{3}} & : c_{3} t_{0} \Rightarrow p_{2} \\ r_{31_{4}} & : c_{4} t_{0} \Rightarrow p_{1} & r_{32_{4}} & : c_{4} t_{0} \Rightarrow p_{2} \\ r_{41} & : p_{1}, p_{2} \Rightarrow \neg v_{1} & r_{42_{1}} & : c_{1} t_{0} \Rightarrow v_{1} \\ & & r_{42_{2}} & : c_{2} t_{0} \Rightarrow v_{1} \\ & & r_{42_{3}} & : c_{3} t_{0} \Rightarrow v_{1} \\ & & r_{42_{4}} & : c_{4} t_{0} \Rightarrow v_{1} \\ r_{51} & : \Rightarrow v_{1} & r_{52} & : \Rightarrow p_{1} \\ & & r_{53} & : \Rightarrow p_{2} \\ r_{31_{1}} & \succ r_{21} & r_{32_{1}} & \succ r_{22} \\ r_{31_{2}} & \succ r_{21} & r_{32_{2}} & \succ r_{22} \\ r_{31_{3}} & \succ r_{21} & r_{32_{3}} & \succ r_{22} \\ r_{31_{4}} & \succ r_{21} & r_{32_{4}} & \succ r_{22} \\ r_{42_{1}} & \succ r_{41} & r_{42_{2}} & \succ r_{41} \\ r_{42_{3}} & \succ r_{41} & r_{42_{4}} & \succ r_{41}\end{array}$

Given that theory, the Reasoner concludes $+\partial \neg l_{1}$, $+\partial \neg l_{2},+\partial \neg l_{3},+\partial p_{1},+\partial p_{2},+\partial v_{1}$ as shown in Fig. 14.

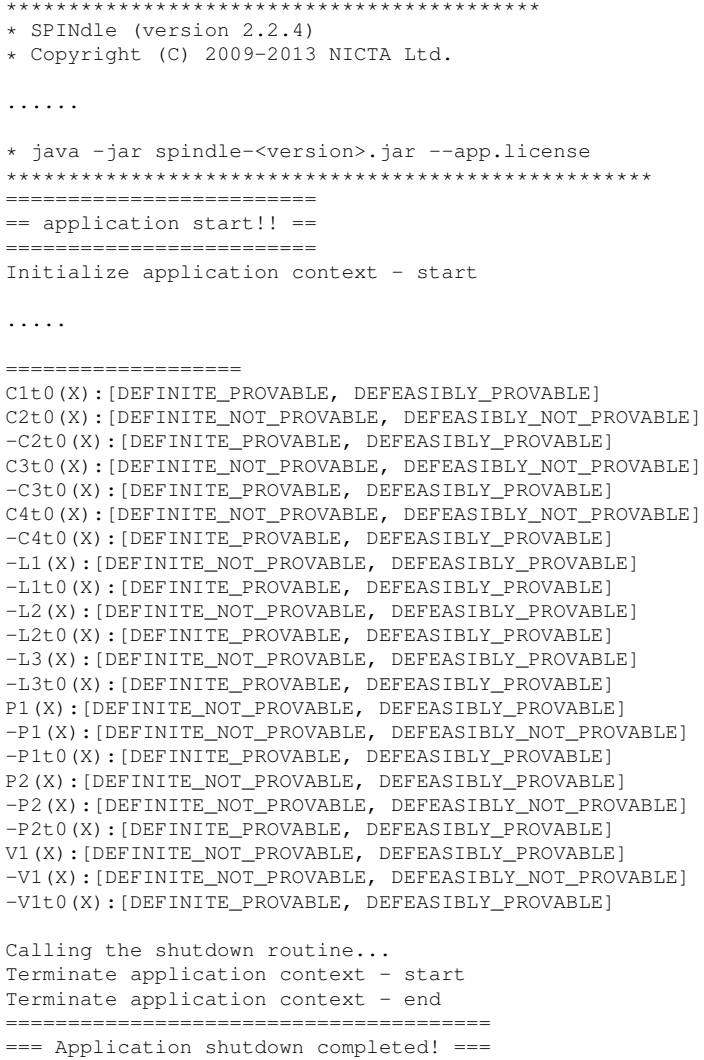

Fig. 14. Spindle conclusions for rules of the reference implementation above

Using these conclusions and acknowledging that no changes are to be made on $c_{1}, c_{2}, c_{3}, c_{4}$ the following will be the desired configuration

$\left\{c_{1}, \sim c_{2}, \sim c_{3}, \sim c_{4}, \sim l_{1}, \sim l_{2}, \sim l_{3}, p_{1}, p_{2}, v_{1}\right\}$

therefore the Actuator leave the lights switched off but will switch on the printers and the coffee machine.

\section{Conclusions and future work}

The efficiency of energy consumption is a pressing and complex problem. One of its main issues is dealing with conflictual rules, that describes the behaviour of devices. The process of specifying rules for energy saving based upon an architecture inspired by non-monotonic reasoning is presented, and studied from the viewpoint of implementation issues. In par- 
ticular, we specify a methodology for translation of rules with temporal and power constraints into defeasible rules. We also provide an architecture proposal for such methodology that implements the mentioned translation method, and actuate the commands as obtained in the translation process.

In the model we have developed, users will specify behavioural rules, which in turn rely on user knowledge and will. Unfortunately, users knowing about energy management and users eager to define rules are quite rare. We therefore can have great benefits if we could somehow suggest a set of behavioural rules which leads to an optimal configuration, a set which can be called "best practices", that can be defined as the problem of extracting the rules of correct behaviour by observing the installation than behaves in the most efficient way, so we have two different sub-problems.

On a general basis, as we have discussed in [16], it is possible to figure out a general method for devising best practice search and comparison. This idea has not yet been implemented in the system fully. The following is a sketch of the further work practices that we are planning out.

First of all we have to find the plant that behaves best among all the ones that are under observation. Unfortunately not all installations are the same: in one home we can have one fridge and forty lamps and one oven, such in a normal flat, while in one other we can have two fridges and two ovens and twenty lamps, such in a house with a garden. Moreover, while in an office we can find several printers and a few coffee machines, in another we can find several coffee machines, a few printers and a lot of table lamps, even though both offices have the same number of computers.

We hence cannot have only one "best" situation, but we will define several "template" plant which will be taken as examples for all similar installations, so we have to define a procedure to define when a plant is similar to another so that they can be considered as belonging to a common group. Which is to say, we have to define a clustering for these installations.

We aim at implementing in a real-life scenario the proposed architecture. Other further developments regard mainly usability issues. In particular, the construction of methods for the specification in visual language of the rules and the creation of a technology for the synthesis of rules from best practices.

Another important step to take is the analysis of a technology that includes rules to govern devices with more that just ON/OFF conditions, namely devices that can have other states (the simplest example is the stand-by state).

\section{Acknowledgments}

The authors gratefully thank Guido Governatori for several hints and suggestions and Real T s.r.l. for technical assistance.

\section{References}

[1] F. Amigoni, N. Gatti, C. Pinciroli, and M. Roveri, What planner for ambient intelligence applications? IEEE Trans. on Systems, Man, and Cybernetics, Part A, 35(1):7-21, 2005.

[2] G. Antoniou, D. Billington, G. Governatori, M.J. Maher, and A. Rock, A family of defeasible reasoning logics and its implementation. In Werner Horn Editor, Proceedings of the 14th European Conference on Artificial Intelligence, ECAI 2000, Berlin, Germany, August 20-25, IOS Press, pp. 459-463, 2000.

[3] G. Antoniou, D. Billington, G. Governatori, and M.J. Maher, Representation results for defeasible logic. ACM Trans. on Computational Logic, 2(2):255-287, 2001.

[4] S. Bahadori, A. Cesta, G. Grisetti, L. Iocchi, R. Leone, D. Nardi, A. Oddi, F. Pecora, and R. Rasconi, Robocare: Pervasive intelligence for the domestic care of the elderly. Intelligenza Artificiale, 1(1):16-21, 2004.

[5] S. Bahadori, A. Cesta, L. Iocchi, R. Leone, D. Nardi, F. Pecora, R. Rasconi, and L. Scozzafava, Towards ambient intelligence for the domestic care of the elderly. In P. Remagnino, G. Foresti, and T. Ellis Editors, Ambient Intelligence: A Novel Paradigm. Springer, 2004.

[6] J. Baliga, R. Ayre, K. Hinton, W.V. Sorin, and R.S. Tucker, Energy consumption in optical ip networks. J. Lightwave Technol., 27(13):2391-2403, 2009.

[7] J. Baliga, K. Hinton, R. Ayre, and R. S. Tucker, Carbon footprint of the internet. Telecommunications J. of Australia, 59(1):5.15.14, 2009.

[8] A. Bikakis, G. Antoniou P. Hassapis, Strategies for contextual reasoning with conflicts in ambient intelligence. Knowl. Inf. Syst., 27(1):45-84, 2011.

[9] M. Breheny, The compact city and transport energy consumption. Trans. of the Institute of British Geographers, 20(1):81$101,1995$.

[10] S. Burmester, H. Giese, J. Niere, M. Tichy, J. Wadsack, R. Wagner, L. Wendehals, and A. Zundorf, Tool integration at the meta-model level within the FUJABA tool suite Int. J. Software Tools Technol. Transfer (STTT), 6:203-218, 2004.

[11] A. Cesta, A. Oddi, and S.F. Smith, A constraint-based method for project scheduling with time windows. J. Heuristics, 8(1):109-136, 2002.

[12] D.J. Cook, G.M. Youngblood, and S.K. Das, A multi-agent approach to controlling a smart environment. In J.C. Augusto, and C.D. Nugent Editors, Designing Smart Homes, The Role of Artificial Intelligence, pp. 165-182, 2006.

[13] M. Cristani, E. Karafili, F. Olivieri, and C. Tomazzoli, Defeasible reasoning about electric consumptions. In L. Barolli, M. Takizawa, T. Enokido, A.J. Jara, and Y. Bocchi Editors, 
Proceedings of the 30th IEEE International Conference on Advanced Information Networking and Applications, IEEE Computer Society, 23-25 March, Crans-Montana, Switzerland, pp. 885-892, 2016.

[14] M. Cristani, E. Karafili, and C. Tomazzoli, Energy saving by ambient intelligence techniques. In L. Barolli, T. Enokido, A. Castiglione, and A. De Santis Editors, Proceedings of the 17th International conference on Network-Based Information Systems (NBiS), CPS IEEE Press, pp. 157-164, 2014.

[15] M. Cristani, E. Karafili, and C. Tomazzoli, Improving energy saving techniques by ambient intelligence scheduling. In L. Barolli, M. Takizawa, F. Xhafa, T. Enokido, and J.H. Park Editors, Procedings of the 29th IEEE International Conference on Advanced Information Networking and Applications, Gwangju, Korea, March 25-27, pp. 324-331, 2015.

[16] M. Cristani, F. Olivieri, and C. Tomazzoli, Automatic synthesis of best practices for energy consumptions In L. Barolli, F. Xhafa, and N. Uchida Editors, Proceedings of the 2016 10th International Conference on Innovative Mobile and Internet Services in Ubiquitous Computing, IMIS , Fukuoka, Japan, IEEE Press, pp. 154-161, 2016.

[17] L. Daniele, P.D. Costa and L.F. Pires, Towards a rule-based approach for context aware applications In A. Pras and Ma. van Sinderen Editors, Proceedings of the 13th Open European Sum mer School and IFIP TC6.6 Workshop, Enschede, The Netherlands, July 18-20, Springer, pp. 33-41, 2007.

[18] P. De Leusse, B. Kwolek, and K. Zielinski, A common interface for multi-rule-engine distributed systems In M. Palmirani, M.O. Shafiq, E. Francesconi, and F. Vitali Editors, Proceedings of the 4th International Rule Challenge, CEUR Workshop Proceedings, vol. 649, paper 11, 2010.

[19] E. de Rezende Francisco, F. Aranha, F. Zambaldi, and R. Goldszmidt, Electricity consumption as a predictor of household income: a spatial statistics approach. In C.A.. Davis, and A.M.V. Monteiro Editors, Proceedings of GEOINFO 2006 - 8th Brazilian Symposium on GeoInformatics, Campos do Jordao (SP), Brazil, November 19-22, Springer Verlag, pp. 155-172, 2006.

[20] X. Fan, W.-D. Weber, and L. A.Barroso, Power provisioning for a warehouse-sized computer. In Dean Tullsen Editor, Proceedings of the 34th annual international symposium on Computer architecture, ACM Proceedings, ISCA '07, New York, NY, USA, pp. 13-23, 2007.

[21] H. Hagras, V. Callaghan, M. Colley, G. Clarke, A. PoundsCornish, and H. Duman, Creating an ambient-intelligence environment using embedded agents. IEEE Intelligent Systems, 19(6): 12-20, 2004

[22] G. Governatori, F. Olivieri, A. Rotolo, S. Scannapieco, and M. Cristani, Picking up the best goal an analytical study in defeasible logic. In L. Morgenstern, P. Stefaneas, F. Levy, A. Wyner, and A. Paschke, P7th International Symposium, RuleML 2013, Seattle, WA, USA, July 11-13, Lecture Notes in Computer Science (including subseries Lecture Notes in Artificial Intelligence and Lecture Notes in Bioinformatics), 8035 LNCS, pp. 99-113, 2013.

[23] G. Governatori, F. Olivieri, A. Rotolo, S. Scannapieco, and M. Cristani, The rationale behind the concept of goal Theory and Practice of Logic Programming, 16(3):296-324, 2016.

[24] N. Jinrui, S. Fengchun, and R. Qinglian, A study of energy management system of electric vehicles. In Proceedings of the 2006 Vehicle Power and Propulsion Conference, VPPC '06,
IEEE Press, pp. 1-6, 2006.

[25] E. Kontopoulos, N. Bassiliades, and G. Antoniou, Deploying defeasible logic rule bases for the semantic web. Data \& Knowledge Engineering 66(1):116-146, 2008.

[26] H. Lam and G. Governatori, Towards a model of uavs navigation in urban canyon through defeasible logic. J. Log. Comput., 23(2):373-395, 2013.

[27] H-P. Lam, and G. Governatori, The making of SPINdle. In G. Governatori, J. Hall, and A. Paschke Editors, Proceedings of the International Workshop on Rules and Rule Markup Languages for the Semantic Web, Springer Verlag, pp. 487-499, 2009

[28] D. Nute, Defeasible logic. In Handbook of Logic in Artificial Intelligence and Logic Programming, volume 3. Oxford University Press, 1987

[29] D. Nute, Z. Hunter, and C. Henderson, Defeasible logic graphs: II. Implementation. Decision Support Syst., 22(3):295-306, 1998.

[30] F. Olivieri, G. Governatori, S. Scannapieco, and M. Cristani, Compliant business process design by declarative specifications. In G. Boella, E. Elkind, B.T.R. Savarimuthu, F. Dignum, M.K. Purvis Editors, Proceedings of the 13th International Conference PRIMA 2013: Principles and Practice of Multi-Agent Systems, Lecture Notes in Computer Science, 8291, pp. 213228. Springer, 2013.

[31] L. Pérez-Lombard, J. Ortiz, and C. Pout, A review on buildings energy consumption information. Energy and Buildings, 40(3):394-398, 2008.

[32] D. Preuveneers, and W. Joosen, Semantic analysis and verification of context-driven adaptive applications in intelligent environments J. of Reliable Intelligent Environments, 2016, 2(2):5373, 2016.

[33] M. Santamouris, N. Papanikolaou, I. Livada, I. Koronakis, C. Georgakis, A. Argiriou, and D.N. Assimakopoulos, On the impact of urban climate on the energy consumption of buildings. Solar Energy, 70(3):201-216, 2001.

[34] S. Seys, and B. Preneel, Power consumption evaluation of efficient digital signature schemes for low power devices. In IEEE International Conference on Wireless And Mobile Computing, Networking And Communications, volume 1, pp. 79-86, 2005.

[35] T.G. Stavropoulos, E. Kontopoulos, N. Bassiliades, J. Argyriou, A. Bikakis, D. Vrakas, and I.P. Vlahavas, Rule-based approaches for energy savings in an ambient intelligence environment. In Pervasive and Mobile Computing, 19:1-23, 2015.

[36] The Climate Group, SMART 2020: Enabling the low carbon economy in the information age. The Climate Group, White Paper [Online], available http: //gesi.org/files/Reports/Smart:202020\%

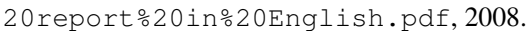

[37] R. S Tucker, R. Parthiban, J. Baliga, Ke. Hinton, R.W.A. Ayre, and W.V. Sorin, Evolution of wdm optical ip networks: A cost and energy perspective. J. Lightwave Technol., 27(3):243-252, 2009.

[38] U.S. Energy Information Administration (EIA), International Energy Outlook 2016., Tech. Rep. DOE/EIA0484(2016), http://www.eia.gov/forecasts/ieo/ pdf/0484(2016).pdf 2016.

[39] G. Wood, and M. Newborough, Dynamic energy-consumption indicators for domestic appliances: environment, behaviour and design. Energy and Buildings, 35(8):821-841, 2003. 